\title{
LA ADJUDICACIÓN JUDICIAL: MODELOS DE RACIONALIDAD JURÍDICA Y PARTICULARI- DADES DE LA DECISIÓN JUDICIAL EN MATERIA DE SALUD
}

\author{
JUDICIAL ADJUDICATION: MODELS OF LEGAL \\ RATIONALITY AND PARTICULARITIES OF THE \\ JUDICIAL DECISION IN HEALTH MATTERS
}

\author{
Lucia Irene Lapenta' \\ Fecha de envío: 05.03.2020 \\ Fecha de aceptación: 10.06.2020
}

DOI: https://doi.org/10.37767/2591-3476(2020)15

RESUMEN:

Este artículo presenta un análisis sobre las particularidades del razonamiento judicial en materia de salud, en el marco de los modelos de racionalidad material. A tal fin, se analizan cinco fallos jurisprudenciales y se ofrecen algunas reflexiones en relación con el razonamien-to judicial subyacente, identificando aquellos elementos que caracterizan la toma de decisión judicial en materia de salud.

ABSTRACT

This article presents an analysis of the specifications in judicial reasoning in health mat-ters, with reference to models of material rationality. To do that, we analyze five jurispruden-tial decisions and offer some reflections in relation to the underlying judicial reasoning, identifying those elements that characterize the judicial decision making regarding the Right to Health.

PALABRAS CLAVE: adjudicación judicial; racionalidad material; Derecho a la Salud.

KEY WORDS: judicial reasoning; material rationality; Right to Health.

\section{Introducción}

El presente artículo se inscribe en el marco del proyecto de investigación titulado "La adjudicación judicial: entre la racionalidad de fines y la racionalidad de necesidades. Un estudio

1 Abogada, Magister en Sociología Jurídica (IISL) y Doctora en Sociología Jurídica (UPV/EHU). Docente en la Facultad de Derecho de Azul (Universidad Nacional del Centro de la Provincia de Buenos Aires). Integran-te del Centro Interdisciplinario de Estudios Políticos, Sociales y Jurídicos (UNICEN). Mail de contacto: luciailapenta@gmail.com. ORCID iD: https://orcid.org/0000-0001-8646-8349. 
de casos de la provincia de Buenos Aires", desarrollado dentro del Centro Interdisciplinario de Estudios Políticos, Sociales y Jurídicos (C.I.E.P.) con inserción en la Facultad de Derecho y la Facultad de Humanas de la Universidad Nacional del Centro de la Provincia de Buenos Aires. ${ }^{2}$ Este proyecto plantea como objetivo central reconstruir los procesos de adjudicación que realizan los jueces, identificando los modelos de racionalidad judicial que subyacen detrás de sus resoluciones. Dentro de este marco, este artículo aborda el análisis de algunas decisiones judiciales en materia de salud.

Si bien es cierto que existe el imperativo general de fundamentar las resoluciones judiciales,$^{3}$ no todas las sentencias evocan una fundamentación igualmente profunda. De acuerdo con los postulados de algunos teóricos como Ronald Dworkin (1975) y Manuel Atienza $(2005,2013)$, cabe distinguir entre aquellos casos fáciles, caracterizados por una cierta claridad sobre las reglas aplicables, sin problemas de interpretación o cuestiones de validez, que resultan por ende más afines a un tipo de razonamiento formal o por subsunción; y por otro lado aquellos otros casos, considerados como casos difíciles, complejos o excepcionales, que conllevan problemas de interpretación, de relevancia, de vacíos legales, de ponderación, y que consecuentemente suelen reclamar otro tipo de razonamiento, con fundamentaciones más profundas y elaboradas. ${ }^{4}$

En este artículo ofrecemos un análisis exploratorio sobre las particularidades de la decisión judicial en materia de salud. Para ello, analizamos cinco casos jurisprudenciales en los que se aplica el derecho a la salud, y ofrecemos algunas reflexiones en relación al razonamiento judicial subyacente. Comenzaremos con un abordaje teórico sobre algunos modelos de racionalidad judicial, donde se incorpora el razonamiento por principios y la tarea de ponderación. Luego, procuraremos ilustrar el mapa de razonamientos y justificaciones subyacentes en los fallos seleccionados. Finalmente, esbozaremos algunas reflexiones integradas a modo de conclusión y plantearemos algunos interrogantes vinculados a la aplicación de una racionalidad de fines en relación al derecho a la salud.

\footnotetext{
2 El núcleo de esta investigación radica en el desarrollo de un análisis profundo sobre el razonamiento judicial detrás de las adjudicaciones judiciales, utilizando para ello resoluciones de sentencia pertene-cientes a diversos casos de la justicia provincial y federal dentro del Departamento Judicial de Azul, Pro-vincia de Buenos Aires, durante el período comprendido entre los años 2008 y 2018.

El criterio de selección de casos presentados en este artículo responde a una distribución pautada in-ternamente entre los miembros del equipo de investigación, en base a un doble criterio: el órgano judi-cial interviniente y las temáticas jurídicas abordadas. Por ello, este artículo recopila exclusivamente aque-llas resoluciones de sentencia emitidas por la Cámara Federal de Apelaciones de Mar del Plata, en rela-ción al derecho a la salud y/o del derecho de las personas con discapacidad, en el período comprendido entre 2008 y 2018.

De acuerdo a estos parámetros, y salvando las limitaciones de acceso a resoluciones digitalizadas en dicho período temporal (cuando la digitalización del trámite judicial no era la regla), los cinco casos que aquí se presentan comprenden todos los fallos dictados por la Cámara en los cuales se observa un desarrollo argumentado de la decisión, más allá de las cuestiones puramente procesales o de forma. El con-junto de casos que aquí se analizan no constituye un número suficiente ni aborda una extensión jurisdic-cional apropiada a los fines de un estudio estadístico y representativo de la jurisprudencia argentina en materia de derecho de salud. Sin embargo, consideramos que constituye una base firme para sostener un primer análisis exploratorio y algunas reflexiones sobre la temática.

3 Cabe mencionar al respecto el artículo 18 de la Constitución Nacional y el artículo 3 del Código Civil y Comercial de la Nación que expresamente dispone "El juez debe resolver los asuntos que sean sometidos a su jurisdicción mediante una decisión razonablemente fundada”. Así también, el artículo 34 inc 4 del Código Procesal Civil y Comercial de la Nación que reza "Son deberes de los jueces: 4. Fundar toda sen-tencia definitiva o interlocutoria, bajo pena de nulidad, respetando la jerarquía de las normas vigentes y el principio de congruencia".

4 Manuel Atienza $(2005,2013)$ incorpora la categoría de casos trágicos, para aquellos casos en los que toda alternativa de decisión judicial supone un daño para algún bien considerado esencial. "Yo creo que en los casos difíciles puede sostenerse, efectivamente, que hay una, una sola, respuesta correcta. Pero que además de casos fáciles y casos difíciles (...) hay casos trágicos: aquellos en los que no es que haya más de una respuesta correcta, sino que no hay ninguna; en los que es imposible realizar la justicia a través del Derecho (...) Los casos trágicos no suponen una claudicación de la razón, de la argumentación. Pero muestran sus límites: los límites de la racionalidad, de la argumentación jurídica" (Atienza, 2013: p.812).

En otro sentido, Miguel Ángel Ciuro Caldani (2005) considera que no existen los casos fáciles, sino que se trata de un fraccionamiento o recorte de la complejidad al momento de construir el caso. "Cada enfoque respecto de los casos, su facilidad y su dificultad, depende de la construcción que se dé al obje-to jurídico (...) El trialismo requiere que el caso, como todo despliegue jurídico, sea construido tridimen-sionalmente como un problema por una adjudicación (...) En la construcción trialista, de una complejidad pura integrada socio-normo-axiológicamente, puede haber casos fáciles y difíciles desde cada una de las dimensiones y consecuentemente en el conjunto, pero nunca al fin sencillos o simples (sin composición), ni siquiera en una de las dimensiones (...) La falta de necesidad de esfuerzo no debe ocultar la compleji-dad de los casos" (Ciuro Caldani, 2005: p.1).
} 


\section{Algunos modelos de razonamiento judicial}

El deber de los órganos judiciales de motivar las resoluciones de sentencia encuentra uno de sus grandes fundamentos en el Estado de Derecho y, en este sentido, opera como garantía propia de todo régimen constitucional de derecho.

La emisión de una sentencia razonablemente fundada implica para los magistrados la aplicación de una cierta racionalidad, en tanto incluye el desarrollo de una argumentación coherente, congruente con las pretensiones y las actuaciones procesales. De este modo, al momento de justificar su decisión, el Juez explicíta la selección y el recorte de los hechos que se han confirmado por las pruebas producidas y los valora junto a las normas jurídicas que considera aplicables al caso. En este sentido, el proceso formativo de la sentencia conlleva indudablemente un momento interpretativo, lógico y valorativo.

Como consecuencia de ello, el motivo o fundamento de una sentencia judicial se constituye con las razones determinantes del mismo acto resolutivo. Allí se exterioriza el razonamiento desarrollado por el órgano que justifica la decisión tomada. No obstante ello, no todas las sentencias contienen una fundamentación de igual profundidad.

El modelo de subsunción es el tipo de razonamiento jurídico más tradicional dentro del ámbito judicial. Este modelo se encuentra guiado por un tipo de racionalidad formal, don-de la seguridad jurídica y la predictibilidad actúan como los valores guía más importantes. Este tipo de razonamiento parte del supuesto que los hechos que son subsumidos en los postula-dos de una determinada norma, y a continuación se obtendrán las consecuencias jurídicas por mera deducción de las premisas.

El razonamiento por subsunción configura el modelo predominante en aquellos casos fáciles, sencillos. Aulis Aarnio describe estos casos rutinarios como aquellos que "consisten en la aplicación de una norma jurídica a un caso individual" (Aarnio, 1991: p.23). El autor los caracteriza en base a dos condiciones principales: por un lado, se trata de casos que se producen reiteradamente, y por el otro, la decisión dentro del caso consiste literalmente en la aplicación de la ley; es decir, que el hecho a determinar depende de la norma jurídica que se aplica, y no de la actividad del operador jurídico que la aplica. De este modo, dentro del esquema de estas decisiones simples, la adjudicación judicial se reduce a una aplicación mecánica de la norma y, por lo general, no queda espacio para la discrecionalidad entre reglas alternativas. ${ }^{5}$

Frente a los casos sencillos se ubican por contraposición los casos difíciles o complejos. Este segundo grupo evoca, a su vez, la aplicación de un tipo de razonamiento particular que se extiende más allá de la mera subsunción, donde se permite desplazar las reglas, a efectos de fundar la resolución judicial en base a principios y valores.

En términos de adjudicación judicial, los casos difíciles implican una decisión discrecional, explica Aarnio, allí cuando más de una norma jurídica puede ser aplicada al mismo conjunto de hechos o cuando la misma norma jurídica permite más de una interpretación (Aarnio, 1991). En este tipo de casos, el decisor actúa acorde con el ordenamiento jurídico, aunque dentro de un cierto margen de discrecionalidad; por lo cual estos casos (a diferencia de las decisiones rutinarias) resultan de alto interés desde el punto de vista de la interpretación del derecho.

5 En consonancia con ello, indica Aarnio, las decisiones rutinarias no resultan de mucho interés desde el punto de vista de la interpretación del derecho (Aarnio, 1991). 
Entonces, en términos de razonamiento judicial, el razonamiento dentro de los casos difíciles excede el modelo tradicional de subsunción. En este sentido, Lars Eriksson (1997) indica la aparición de nuevos tipos de razonamiento jurídico (o modelos de argumentación jurídica), con motivo de una cierta mutación del derecho moderno hacia tendencias conflictivas. ${ }^{6}$ Eriksson sostiene que la función del derecho moderno es la de reforzar un orden y resolver conflictos: "el sistema jurídico es, en otras palabras, un orden de integración y un orden de resolución de conflictos" (Eriksson, 1997: p.114). No obstante ello, el autor indica que esta función de orden se encuentra en crisis y expresa que "una característica esencial del derecho moderno es que no existe un principio unificador que garantice la coherencia del sistema" (Eriksson, 1997: p.113).

Entonces, según Eriksson, aquel orden jurídico que estaba guiado por una racionali-dad formal, por normas generales y abstractas, es visto hoy como un derecho que se acerca hacia otros modelos de racionalidades materiales, conformadas por "valores políticos, económicos, sociales y morales que se construyen dentro del orden jurídico, de modo que la toma de decisión jurídica se parece cada vez más a la toma de decisión política y los economistas, sociólogos, asistentes sociales y tecnócratas comienzan a tener una influencia cre-ciente en la aplicación práctica del derecho" (Eriksson, 1997: p.115).

Lars Eriksson enfatiza sobre el surgimiento de dos tipos de racionalidades en particular: la racionalidad de fines y la racionalidad de intereses. Según el autor, ambos modelos de razonamiento o argumentación jurídica coexisten en el orden jurídico de hoy. ${ }^{7}$

En el modelo de racionalidad de fines, "las reglas jurídicas y los principios que las respaldan le proporcionan al juez aquellos fines e intereses que funcionan como premisas básicas de la argumentación (...) Dentro del marco de lo factible y aceptable, el juez puede tomar sus decisiones de un modo comparativamente irrestricto y permitir que el problema mismo determine la decisión final" (Eriksson, 1997: p. 121). En otras palabras, el modelo racional de fines adopta una mirada más sistémica y la racionalidad de la decisión tomada se mide en función de lograr una armonía entre los intereses en conflicto, a través de una decisión socialmente equilibrada.

De modo que no se trata de un modelo deductivo de subsunción, sino que existe una cierta elaboración por parte del juez en miras al sistema y a la sociedad en su conjunto al momento de fundar la resolución judicial. Así también, a diferencia del modelo de subsunción, en este modelo de racionalidad el hecho a determinar no surge de la regla misma, sino más bien de una conjunción coherente entre los hechos del caso, la norma y los fines últimos del sistema normativo.

El papel del juez dentro del modelo racional de fines es mucho más activo que el de un

\footnotetext{
6 El autor plantea el desarrollo de ciertas tendencias conflictivas dentro del derecho moderno y puntualiza sobre la existencia de dos fenómenos crecientes: la "materialización" y la "hiperjuridización".

La creciente materialización consiste en el pase de un orden jurídico guiado por una racionalidad formal hacia una racionalidad material, compuesta por valores políticos, económicos, sociales y morales que se construyen dentro del propio orden jurídico. La hiperjuridización se presenta en tanto cada vez más ciertos aspectos que no estaban regulados por el Derecho pasan a ser regulados y en consecuencia asumen luego el carácter de conflictos justiciables. De este modo, según el autor, los órdenes (económi-co, cultural, simbólico, político) que antes eran autónomos, están ahora sometidos a una intensa regulación jurídica.

7 Jeremy Waldron (2000) presenta un argumento similar en un artículo posterior cuando refiere al lengua-je de los derechos y el lenguaje de las necesidades. En su artículo observa que tipo de lenguaje se utiliza por parte del peticionante a la hora de realizar el reclamo y determina como no sólo resultan complementarios sino que conviene una utilización conjunta en tanto el lenguaje de los derechos le otorga un marco de firmeza y responsabilidad al lenguaje de las necesidades.
} 
mero aplicador. Su rol comprende la tarea de interferir la realidad mediante correctivos y de conjeturar a través de su propia resolución judicial en base a una racionalidad sustancial guiada por los fines expresados en las normas.

Por otro lado, el modelo de racionalidad de necesidades se considera, según Eriksson, como una variante de la racionalidad de fines. Este modelo se desarrolla "como resultado de la legislación propia del Estado Social de Bienestar, la cual atiende a las necesidades concretas y reales de los miembros de la sociedad de un modo mucho más amplio que las legislaciones anteriores" (Eriksson, 1997: p.121). Dentro de este modelo, la racionalidad de la decisión (es decir, la validez de los argumentos) se mide por sus efectos en las necesidades concretas de las personas involucradas en el caso y la capacidad de la resolución judicial para satisfacer dichas necesidades.

A modo de comparación, podemos decir que el modelo racional de fines busca decisiones socialmente equilibradas, es decir, que propende a generar un cierto orden con base en decisiones proyectadas dentro de un marco sistémico mayor. Por el contrario, el modelo racional de necesidades busca recuperar o restaurar un cierto orden afectado mediante la satisfacción óptima de las necesidades concretas de las personas involucradas en el caso.

A nuestro entender, de la mano de estos dos modelos de racionalidad, entra en juego también el cambio en la concepción de las normas y el modo de entender la aplicación del derecho. Es decir, que resulta posible establecer una vinculación estrecha entre estos tipos de racionalidades materiales y la distinción dentro del complejo de las normas jurídicas entre las reglas jurídicas, por un lado, y los principios normativos, por el otro. Ofrecemos mayores precisiones sobre esta cuestión en el próximo apartado.

\section{El lugar de los principios dentro de la adjudicación judicial}

Para Robert Alexy (2001), los principios son normas que ordenan que se realice algo en la mayor medida posible. Los principios son, por consiguiente, mandatos de optimización que se caracterizan porque pueden ser cumplidos en diversos grados, en tanto la medida que ordenan cumplir depende de las posibilidades fácticas y también de las posibilidades jurídicas.

En este sentido, los principios así comprendidos también se enrolan dentro del proceso de materialización del orden jurídico en cuanto marcan la introducción de valores e intereses. Éstos funcionan esencialmente como lazos entre el sistema jurídico y sistemas u órdenes no jurídicos, como la moral y la política. ${ }^{8}$

En el marco de este trabajo, cabe indagar sobre cuál es la lógica y el tipo de razona-miento que se esconde detrás de los principios. Lars Eriksson indica que "Los principios son capaces de proporcionar al menos una coherencia fragmentaria a los sistemas parciales del orden jurídico. La función primaria de los principios substanciales es, precisamente, conferir estabilidad a dichos sistemas, funcionando como medios para la sistematización e interpretación de las reglas jurídicas" (Eriksson, 1997: p.119). ${ }^{9}$

8 La vinculación existente entre Derecho y Moral, según Alexy, resulta más clara en los principios constitu-cionales, como los de dignidad humana, libertad, igualdad, democracia, Estado de derecho y Estado social.

9 Para Eriksson "una característica esencial del derecho moderno es que no existe un principio unificador que garantice la coherencia del sistema”; “... el orden jurídico moderno debe, pues, unificar principios integradores incompatibles...” (Eriksson, 1997: p. 113/115). Aparece en estas citas la referencia 
Recordamos que el modelo racional de la subsunción presenta la aplicación del Derecho como un proceso que consiste en la determinación mecánica de las consecuencias ya previstas para los hechos concretos. Desde la mecánica de la subsunción, la operación de justificación se agota en mostrar que el supuesto de hecho real coincide con el supuesto normativo previsto por el legislador.

En la actualidad, la creciente referencia a los principios ha supuesto un nuevo modo de entender la tarea de aplicación del Derecho, ubicada muy lejos de lo que podamos imaginar como una tarea mecánica. Uno de los primeros motivos que explica esta distancia con la aplicación mecánica radica en el hecho de que el universo de los principios introduce una categoría de principios normativos donde el significado no es evidente o no se encuentra absolutamente determinado. Así, se contrapone la cualidad de determinación presente en las reglas y la propuesta de optimización que introducen los principios.

Las reglas tienen una determinación precisa del supuesto de hecho y permiten una aplicación por subsunción y deducción (Aarnio, 1991). Los conflictos entre reglas, también llamados antinomias, se resuelven por razones de jerarquía, temporalidad o especialidad; más nunca puede existir una aplicación dual en el universo de las reglas jurídicas.

Por el contrario, los principios siempre incluyen un cierto grado de indeterminación. Éstos son indeterminados porque no tiene un supuesto de hecho específico, sino que se plantean como guías para el razonamiento legal (Aarnio, 1991). De este modo, resulta imposible pensar en la deducción o subsunción de los hechos dentro de un principio normativo. Sumado a ello, a diferencia de las antinomias, puede existir una aplicación dual en el universo de los principios y resulta incluso factible que estos dos (o más) principios se encuentren en contraposición.

Dentro del esquema de los principios, la colisión entre ellos no conlleva una opción de alternativas excluyentes, sino que se origina una tarea de ponderación de principios a cargo del juez que atiende el caso concreto. Toda ponderación o medición de la importancia de los principios deriva en la construcción de una "jerarquía axiológica o móvil" (Guastini, 2001), entendida como un orden de preferencia mutable y dependiente de cada caso concreto. ${ }^{10}$

La tarea de la ponderación de principios, al igual que la subsunción, constituye una instancia de construcción argumental. Es cierto que ambos parten de puntos de inicio diferentes y que se orientan en direcciones opuestas. Aún así, en ambos casos se encuentran presente la necesidad de esclarecer el problema jurídico, de ofrecer claridad en la exposición y de resguardar una cierta coherencia lógica, de buscar solidez en la argumentación, de mantener una congruencia con el desarrollo del caso en el marco del proceso judicial, etc. De este modo, más allá de estas condiciones comunes (que podríamos llamar precondiciones de todo modelo de racionalidad), entendemos que los principios se apartan del modelo de la racionalidad formal o la mecánica de la subsunción. Por el

\footnotetext{
a la tarea de ponderación de principios que encabezan los jueces en el derecho actual. Consideramos que el inte-rrogante que surge en este contexto de incompatibilidades es si existe la unidad del orden jurídico hoy, si resulta ello deseable y, en todo caso, cómo garantizar esa moción de unidad dentro del derecho.

10 En el mismo sentido, Gustavo Zagrebelsky (2003) refiere a un "derecho dúctil", en cuanto a aquel orde-namiento jurídico que, informado por principios de justicia material, resulta maleable en la medida en que ningún principio prevalece en forma predeterminada sobre el otro. La prevalencia de los principios se evalúa y se establece dentro de cada caso concreto. Así explica el autor que, al momento de la ponde-ración, el juez evalúa si el resultado que produciría la aplicación de un principio en el caso concreto le parece más justo (o menos injusto) que el resultado que derivaría de aplicar el segundo principio.
} 
contrario, éstos mantienen una clara cercanía con los modelos de racionalidad material $y$, en particular, con los modelos de racionalidad de fines y de necesidades que plantea Lars Eriksson. Comparten entre sí las bases de la vinculación material y sustantiva dentro del orden normativo, que conforman nuestro Derecho actual, en equiparación a las condiciones de taxatividad y determinación propias de las reglas jurídicas. ${ }^{11}$

\section{Análisis de casos en materia de salud}

En este apartado realizamos el análisis de cinco sentencias de la Cámara Federal de Apelaciones de Mar del Plata, todas ellas referidas al Derecho a la Salud. En términos generales, todos los casos encuentran su origen en un reclamo individual frente a obras sociales (públicas y privadas), y se vinculan con la aplicación de la Ley de Obras Sociales (ley nro. 23660), Ley del Sistema Nacional de Seguro de Salud (ley nro. 23661), Ley de Prestaciones Médicas (ley nro. 26682) y Ley de Discapacidad (leyes nro. 24901 y 22431). A su vez, desde el punto de vista de la petición, en todos los casos se encuentra alguno de los siguientes patrones: el reclamo ante la negativa expresa de cobertura médica o el silencio arbitrario de la obra social ante el pedido del beneficiario; o el reclamo por limitaciones en la cobertura médica (en particular, la derivación con un especialista determinado).

Sentados estos rasgos generales, nos interesa plasmar algunos elementos particulares de cada caso y a continuación ofrecemos un análisis integrado de todos ellos en términos de razonamiento judicial y de aplicación de principios.

\section{1. "García Ivana R. c/ C.S. Salud s/amparo"}

En el caso "García Ivana R. c/ C.S. Salud s/amparo" causa nro. 13330, con sentencia de la Cámara del año 2012, se presenta la negativa de la obra social a cubrir una intervención quirúrgica de By Pass gástrico requerido por la actora. La sentencia de primera instancia decla-ra arbitraria la omisión de la demandada y la obliga a brindar una cobertura integral. En segunda instancia se confirma esta decisión. Cabe analizar aquí los argumentos expuestos por el Tribunal y el razonamiento desarrollado en el caso concreto.

En oportunidad de la expresión de agravios, la obra social alude a la inexistencia de un accionar ilegítimo o arbitrario, considerando el marco contractual que rige su vínculo con la beneficiaria del servicio de salud. A su vez, se indica que la actora no habría cumplido con sus obligación de combatir la obesidad a través de métodos no invasivos (recaudo que exige la normativa de la obra social para este tipo de diagnóstico).

Dentro de los elementos de mayor interés, se destaca la alusión de la parte agraviada a la vulneración del principio de división de poderes, al considerar que la sentencia dictada en primera instancia constituye una interferencia indebida en la esfera del Poder Legislativo (órbita del Ministerio de Salud), en tanto se ordena la obligatoriedad o no de la prestación de prácticas médicas. ${ }^{12}$ Resulta interesante en este caso notar como los argumentos de la expre-sión de agravios de las partes influyen (más no limitan) sobre el razonamiento posterior del Tribunal.

\footnotetext{
11 No existe una exclusión entre principios y reglas, sino una relación de simbiosis complementaria. Ante la insuficiencia de las reglas, el Derecho precisa de principios y, a su vez, estos requieren de las reglas para su mejor optimización (Aarnio, Alexy, Zagrebelsky).

12 Consideramos que la referencia a la vulneración de este principio plantea un punto interesante en tér-minos de adjudicación judicial, en relación a la extensión del ámbito normal o legítimo de las facultades del juez al momento de resolver casos donde se encuentran vulnerados derechos fundamentales. En otras palabras, ¿¿hasta qué punto puede el juez extenderse en su tarea de interpretación acorde a las normas y lineamientos normativos en el ámbito del derecho a la salud y la vida? Retomaremos este punto en el apartado de reflexiones finales.
} 
En términos de razonamiento judicial, la Cámara apela expresamente a un razona-miento por necesidades indicando que "debe buscarse una solución que 'fundada en dere-cho' satisfaga de la mejor manera posible 'la necesidad de la amparista' de poner en resguardo su derecho a la salud" (Consid. III, Voto Dr. Tazza). A su vez, pone en consideración la situación de salud de la actora, enlistando sus antecedentes patológicos, las consecuencias de su enfermedad (obesidad mórbida grado IV), e indicando así también la necesidad de conside-rar el deterioro en su calidad de vida como una variable a analizar.

En cuanto a la valoración de los elementos probatorios, vinculados a los intentos con procedimientos alternativos, el Tribunal indicó que "el evidente fracaso del tratamiento no es un dato menor a la hora de decidir si la prestación reclamada es la adecuada y si la accionante ha logrado acreditar el cumplimiento de los recaudos exigidos por la reglamentación" (Consid. III, Voto Dr. Tazza). El Tribunal transparenta aquí la dirección de su razonamiento dirigido a determinar la adecuación o no de la prestación reclamada por la actora. La prueba parece ser incorporada como elemento complementario, más no central, dentro de la línea de razona-miento.

Dentro de los fundamentos normativos, el Tribunal indica la vigencia de la Ley 26396 (Ley de Prevención y Control de los Trastornos alimentarios), legislación que incorpora al Programa Médico Obligatorio (PMO) la cobertura del tratamiento integral de los trastornos alimentarios. A raíz de ello, el Tribunal reafirma por derivación normativa, la existencia de la obligación a cargo de la obra social de ofrecer la cobertura reclamada. Aquí las disposiciones normativas, al igual que el material probatorio, complementan el proceso de razonamiento de la Cámara de Apelaciones.

\section{2. "Gastón José Luis y otros c/OSDE Binario s/Amparo Ley"}

En el caso "Gastón José Luis y otros C/OSDE Binario s/Amparo Ley" causa nro. 1925/2013, con sentencia de la Cámara del año 2014, se presenta el reclamo frente a una orden de la obra social de cambiar de especialista, encontrándose la paciente con el tratamiento en curso.

La afectada es una menor de edad discapacitada que padece un "trastorno generalizado del desarrollo" (TGD) que afecta su sistema neurológico, particularmente en el área del lenguaje. Se encontraba desarrollando una terapia en etapa de diagnóstico con una especialista en neurolingüística fuera de su ciudad de origen y, finalizado el diagnóstico, fue derivada a una terapia especial (abordaje terapéutico del modelo DIR y estrategias de la técnica PROMPT) con la misma profesional. Frente a ello, la obra social ofrece la cobertura médica de sesiones con una fonoaudióloga en su ciudad de residencia. Esta circunstancia implicaba obligar a la paciente (frente a la falta de cobertura) a un cambio de la terapia en curso y al cambio de especialista.

Frente a estos hechos, la sentencia de primera instancia rechaza el reclamo de la actora, por considerar que la obra social requerida tiene la obligación de proveer un especialista para atender a sus afiliados, pero aun así no se encuentra obligada a proveer uno en particular. En segunda instancia se revoca la decisión de origen ordenando a la obra social brindar una cobertura completa del tratamiento especializado con la Licenciada de confianza de la actora.

En este caso, el Tribunal desarrolla explícitamente un tipo de razonamiento por nece- 
sidades, afirmando que "para evaluar la viabilidad jurídica de las pretensiones de las partes es esencial el análisis de las circunstancias específicas del caso" (Consid. VI Voto Dr. jiménez). Se plantea la necesidad de resolver sobre un conflicto general-particular, que se presenta entre el requerimiento objetivo de toda obra social de prestar cobertura y la necesidad planteada en el caso concreto de continuar con una determinada profesional.

Dentro de los elementos de interés, se destaca de forma notoria la consideración por parte del Tribunal del lazo terapéutico y afectivo (en términos de confianza) ya generado entre la menor de edad y la especialista a lo largo de del tratamiento en curso. ${ }^{13}$ " $\ldots$ la presta-dora carecía de motivo para objetar la idoneidad de la Licenciada Villa, quien es la profesional tratante de la niña, en su patología grave que padece desde su nacimiento, teniendo que prevalecer en estos casos singulares no sólo la mentada idoneidad del profesional, sino tam-bién la relación médico-paciente que en estos casos resulta de trascendental significado, ya que debido a la enfermedad que padece, es de consecuencias imprevisibles cambiar de galeno y tratar con un profesional que desconoce totalmente su cuadro..." (Consid. VI Voto Dr. Jiménez) (el destacado me pertenece).

A nuestro entender, resulta interesante ahondar sobre el significado de esos "casos singulares" que queda omiso dentro del fallo. ¿Cuál es ese universo de casos? ¿Refiere el Tribunal a los casos con tratamiento en curso? ¿Serán los casos con tratamiento en curso y buenos resultados? ¿Referirá a los casos con este tipo de padecimiento neurológico?

Dentro de los fundamentos normativos, el Tribunal indica que "si bien es cierto que la Ley 24901 no contempla la cobertura de todos aquellos requerimientos que efectúen las personas con discapacidad, prioriza aquellos considerados 'más aptos' para cubrir las necesidades de salud del reclamante, y enfatiza la necesidad de resguardar la relación médico-paciente, cuando ella se encuentra afianzada" (Consid. IV, Voto Dr. Jiménez). Del mismo modo, resulta aquí complejo determinar cuándo es posible considerar que existe una relación afianzada.

Al momento de analizar el accionar de la obra social, la Cámara indica que "el hecho de haber ofrecido una cobertura alternativa a la requerida por la actora no obsta en modo alguno a la existencia de un acto arbitrario" (Consid. VI, Voto Dr. Jiménez). Asimismo, se destaca que el incumplimiento de OSDE se "agrava" teniendo en cuenta "la gran vulnerabilidad de la peticionante de autos (niña con discapacidad)".

Por último, el Tribunal destaca la obligación de justificación en las omisiones de prestaciones médicas obligatorias. De este modo, la Cámara califica el accionar de la obra social como una omisión arbitraria, en tanto existió una omisión sin expresión de justas razones o motivos. Aún más, se destaca una cierta finalidad sistemática al indicar que un acto se torna arbitrario cuando "no obstante el eventual apoyo legal, produce, dentro del marco de la ley, efectos contrarios a los previstos, violándose garantías constitucionales" (Consid. IV, Voto Dr. Jiménez).

“Además es el artículo 14 bis de la Constitución Nacional el que establece que ‘el estado otorgará los beneficios de la seguridad social que tendrá carácter de integral e irrenun-

$13 \mathrm{Al}$ momento de la expresión de agravios, la actora hace hincapié en la confianza como elemento primor-dial para la buena marcha del tratamiento. Se indica que el tratamiento ya comenzado había ofrecido resultados satisfactorios, y que el hecho de cambiar de profesional implicaría un retroceso en la evolu-ción de la paciente. 
ciable'. Este principio, entonces, es el que debe guiar este decisorio, y sus excepciones deben encontrarse razonablemente justificadas no sólo en la normativa aplicable, sino también frente al caso en concreto" (Consid. VI Voto Dr. Jiménez). De este modo, continuando con el razonamiento por necesidades, la Cámara reconsidera la calificación de la arbitrariedad (en el marco de un proceso de amparo) en vinculación a la vulneración de los intereses en el caso concreto, más allá de la normativa aplicable.

\section{3. "Muñoz José Alberto c/INSSJP y otros s/ Amparo"}

En el caso "Muñoz José Alberto c/INSSJP y otros s/ Amparo", causa nro. 3957/2013, con sentencia de la Cámara del año 2014, se presenta el reclamo judicial frente al rechazo de cobertura médica de una internación en la "Posada del Inti", una institución especializada en adicciones. Este caso llega a instancia de la Cámara Federal a través de la apelación por parte de la obra social contra la resolución que hace lugar a la medida cautelar, ordenando la cobertura completa de la internación en favor del beneficiario discapacitado.

Al momento de expresar agravios, se invoca también la vigencia del principio de divi-sión de poderes, criticando la ausencia de fundamento legal de órgano judicial para ordenar al Ministerio de Salud medidas que resultan totalmente ajenas a la órbita de competencia judicial.

Dentro de la resolución, el Tribunal analiza los derechos en juego y hace hincapié en el carácter absoluto de los derechos implicados en el caso. “Debo valorar la trascendencia de los derechos que se encuentran comprometidos en autos, así el derecho a la vida, a la Salud y a una asistencia médica adecuada" (Voto Dr. Tazza). Se hace referencia al carácter absoluto del derecho a la inviolabilidad de la vida, a una buena calidad de vida y por ende a una adecuada atención médica, como centro del Sistema de Derechos Humanos. Asimismo, se hace men-ción a la contracara del derecho a la vida, que constituye una obligación activamente universal en cabeza de los Estados, como un deber de hacer positivo.

A continuación, la Cámara realiza una evaluación entre varios elementos fácticos, ra-zonamiento dentro del cual incorpora la condición de salud de la paciente, los padecimientos previos, su condición de discapacidad, el peligro de la demora (tanto en términos sustanciales de salud, como procesales de la medida cautelar solicitada) y así también la condición cautelar (y no definitiva o de fondo) de la decisión judicial a tomar en el caso.

Queda en evidencia dentro del hilo argumental del Tribunal, la búsqueda de una solución de justicia para el caso concreto, en particular cuando toma en cuenta los padecimientos previos y concluye que "no corresponde agravar más aún sus condiciones" (Voto Dr. Tazza), intentando evitar un perjuicio irreparable o de difícil solución.

\section{4. "P.A.R. y otro c/Construir Salud s/Ley de Incapacidad"}

El caso "P.A.R. y otro c/Construir Salud s/Ley de Incapacidad", causa nro. 27894/2016, con sentencia de la Cámara del año 2017, se presenta un reclamo por el rechazo de la obra social a entregar una medicación importada (aceite de cannabis), gestionada debidamente a través de la Administración Nacional de Medicamentos, Alimentos y Tecnología Médica (AN-MAT).

El paciente implicado en el caso sufre de epilepsia y retraso mental, y presenta su reclamo en virtud de la Ley de Discapacidad. La resolución de primera instancia rechaza la 
medi-da cautelar por la cual se solicitaba la entrega del medicamento, con fundamento en la falta de reglamentación de la Ley 27350 (Ley de Investigación médica y científica del Uso medicinal de la planta de Cannabis y sus derivados). ${ }^{14}$ En segunda instancia se revoca la decisión apelada y se le ordena a la obra social hacer entrega inmediata del medicamento al beneficiario.

Al momento de resolver, el Tribunal hace hincapié en el carácter absoluto de los derechos en juego, el derecho a la inviolabilidad de la vida, a una buena calidad de vida, considerados como centro del Sistema de Derechos Humanos. A su vez, en vista de lo resuelto en instancia de origen, la Cámara de Apelaciones incorpora argumentos en torno a la condición de normas operativas y programáticas cuando se trata particularmente de la recepción de derechos fundamentales. El Tribunal sostiene al respecto la presunción de que las normas dictadas en materia de derechos humanos son operativas.

Luego, se presentan también algunos argumentos que denotan la aplicación de un razonamiento por necesidades, en tanto se refiere a las circunstancias del caso particular y se destaca de manera especial la vulnerabilidad del paciente. "En el caso de marras, he de pon-derar la patología que aqueja al amparado, su condición de discapacitado y la prescripción efectuada por el médico tratante que desarrolla su actividad en una institución de reconocido prestigio e indudable jerarquía científica, como lo es la Fundación FLENI." (Voto Dr. Ferro).

Además, se incluye dentro del hilo argumental la referencia al tiempo como un ele-mento determinante, en términos de perjuicio para la salud y en términos procesales o de demora en el tiempo. "Todo ello, sin dejar de advertir el largo tiempo que ha transcurrido desde el inicio de la presente acción y el momento en que el tema referente al rechazo de la medida cautelar es revisada por esta Alzada" (Voto Dr. Ferro) (el destacado me pertenece).

Sumado a ello, en reiteradas ocasiones se incorpora dentro de los considerandos del fallo la referencia al criterio médico, entendido como criterio de autoridad, que opera dentro de la resolución como un argumento de refuerzo. "El certificado de discapacidad agregado y la evidente urgencia que demandada la necesidad para el paciente de disponer de un tratamiento adyuvante paliativo (aceite de cannabis) (...) indicado por el profesional médico tratante constituye suficiente fundamento para tener por acreditado el peligro en la demora y la verosimilitud del derecho que impone el dictado de una medida cautelar" (Voto Dr. Ferro) (el resaltado me pertenece).

Por las afirmaciones vertidas a lo largo de los distintos considerandos, se puede dedu-cir un intento de asimilar y combinar los dos tipos de racionalidad (de fines y de necesidades), aparentemente armónicas entre sí en el caso en cuestión. Así, la Cámara refiere a que en el marco de una medida cautelar "el juez posee amplias facultades para proceder al análisis de los hechos así como para valorar los intereses de las partes, no encontrándose vinculado por la petición que se le formule al respecto, quedando librado a su prudente arbitrio resolver lo que sea más razonable para satisfacer aquellos intereses y los más generales y preferentes del servicio de justicia" (Voto Dr. Ferro) (el resaltado me pertenece).

$14 \mathrm{El}$ juez de primera instancia se escuda en parte en cierto vacío normativo existente, arguyendo la falta de creación del Registro dispuesto por dicha legislación como así también la falta de reglamentación acerca de la autoridad de contralor correspondiente. Sin ahondar mucho más en los argumentos, es posible notar a simple vista la aplicación del modelo de subsunción en esta resolución de origen. 
Sin embargo, al momento del cierre de los argumentos, el Tribunal hace explícita la fundamentación del decisorio en base a las necesidades del peticionante, afirmando que “Teniendo en cuenta pues las dolencias que padece el tutelado, es indudable que requiere de un pronto remedio, o dicho en términos constitucionales 'de una acción positiva' que le asegure la vigencia del derecho a la salud, dentro de la garantía constitucional a una tutela judicial continua y efectiva" (Voto Dr. Ferro).

\section{5. "F.R. c/INSSJYP s/Ley de Discapacidad"}

En el caso "F.R. c/INSSJYP s/Ley de Discapacidad", causa nro. 481/2014, con sentencia de la Cámara del año 2015, se presenta un reclamo por el silencio arbitrario de la obra social frente a un pedido de cobertura de servicio de enfermería 24 hs. para un paciente de 70 años, que sufre de cuadriplejia y ACV agudo. La sentencia de primera instancia condena a la obra social a prestar el servicio en forma completa. En segunda instancia se confirma la resolución de origen, con algunos elementos y razonamientos particulares que cabe destacar aquí.

A lo largo de la resolución, la Cámara destaca varios elementos particulares en relación a la persona del peticionante que incorpora como argumentos para reforzar la decisión. Así, por ejemplo, el Tribunal indica expresamente la condición de vulnerabilidad cuando indica que "el afiliado promoviente padece a las claras de una 'doble vulnerabilidad', ya que este anciano resulta ser una persona con discapacidad" (Voto Dr. Jiménez).

La Cámara refiere expresamente a la existencia de una discriminación o "desigualdad material de los ciudadanos ancianos", categoría que identifica como mayores de 65 años, jubi-lados y pensionados. El Tribunal expresa que esta desigualdad resulta particularmente visible relación a las prestaciones de salud.

Luego, se incorpora también una mención sobre la situación económica del paciente como elemento de peso dentro del análisis: "se encuentra fuera de discusión la avanzada edad del actor, su delicado estado de salud, y su precaria situación económica" (Voto Dr. Ferro). ${ }^{15}$

Respecto al accionar de la obra social, la Cámara reitera que toda omisión en la cobertura médica debe encontrarse razonablemente justificada. Se establece que en este caso "al mantener un silencio arbitrario frente a la solicitud de cobertura de asistencia y cuidado que le fue prescripta, tal actitud de la accionada es injustificada, arbitraria, ausente de motivación y violatoria de las normas en cuestión" (Voto Dr. Ferro).

En relación a la obligación prestacional, la Cámara de Apelaciones refiere al argumento de la integralidad en las prestaciones de salud, considerando que la obra social carga con la obligación de ofrecer el mejor nivel de calidad de prestaciones disponible, y que ello incluye la exigencia de cumplir con las prestaciones establecidas por el Programa Médico Obligatorio.

Por último, se destaca en este fallo particular la vinculación que realizan los Dres. Ferro y Jiménez entre el Derecho de la Salud y Derecho de la Ancianidad, ilustrando en en prác-

15 La Cámara de Apelaciones indica la necesidad de poner en consideración frente a la solicitud de cober-tura en el caso concreto los siguientes elementos: el estado general de salud, el contexto personal del afectado, el contexto familiar y económico del peticionante y la indicación médica que marca el tratamiento idóneo para el paciente. Todos ellos constituyen, según el trabajo doctrinal citado en el fallo, la "trama inescindible" del perfil conceptual del anciano (Morello, Augusto "Las Edades de la Persona en el cambiante mundo del Derecho", Editorial Hammurabi, p. 162). 
tica la complejidad del mundo jurídico y afirmando la necesidad de atender a las nuevas ramas transversales del Derecho. ${ }^{16}$

Se reservan mayores detalles para el análisis integrado de los casos que presentamos a continuación.

\section{Análisis integrado de los casos en estudio}

En términos generales, los cinco casos en estudio son sentencias devenidas en procesos sumarios (o de amparo) vinculados al Derecho a la Salud. Las sentencias corresponden a los años 2012, 2014, 2015 y 2017. Se trata de casos que comprenden prestaciones médicas diversas, desde prácticas de intervención quirúrgica hasta provisión de medicamentos especiales o de servicios de enfermería. A su vez, los reclamos se plantean contra obras sociales del ámbito público y privado.

Partiendo de la consideración de estas características diversas, nos interesa plantear en este punto algunos parámetros generales obtenidos del conjunto de casos, en relación al razonamiento judicial. Se hará hincapié en algunos elementos comunes que son considerados dentro del hilo argumental en todos los casos y, finalmente, se intentará traducir estos elementos utilizados dentro del razonamiento en la aplicación de determinados principios, que actúan como fundamento normativo dentro del decisorio.

En todos los casos, el relato de la sentencia comienza por una síntesis de los hechos y de los argumentos vertidos en los escritos de agravios. Luego, en todos ellos se encuentra presente el análisis de los derechos vulnerados con expresa mención al derecho a la salud, derecho a la vida y derechos de la persona en general.

A nuestro entender, queda claro que los casos en estudio se ubican dentro de la categoría de "casos complejos", donde el Tribunal se aparta de la mera subsunción del caso en la norma y donde desarrolla un mayor esfuerzo en el sostén argumentativo. Además, resulta visible la aplicación de una racionalidad de necesidades, en cuanto se considera esencial el análisis de las circunstancias específicas de cada caso concreto.

Dentro de estas consideraciones generales, nos interesa enlistar (sin ningún tipo de orden jerárquico) ciertos elementos peculiares que inciden en el razonamiento judicial en materia de salud.

Primer elemento: el tiempo.

El tiempo se encuentra presente en estos casos, tanto en términos de tiempo proce-sal como en términos de pronóstico de salud. A modo de ejemplo, se argumenta que "... ante un padecimiento gravísimo como el que se acredita en autos, se impone una rápida auditoría, que no conlleve dilaciones como las advertidas en autos. O sea, no se trata -como pretende simplificar la prestadora- de judicializar la cobertura con un profesional determinado, sino de proveerlo y auditarlo con la celeridad que el caso amerita"... (Caso Gastón, José Luis y otros c/OSDE, Consid. VI, Voto Dr. Jiménez). 
Así también, se expresa que “...teniendo en cuenta pues las dolencias que padece el tutelado, es indudable que requiere de un pronto remedio..." (Caso P.A.R. y otro c/Construir Salud, Voto Dr. Ferro).

A nuestro entender, esta consideración del elemento tiempo se traduce dentro de la argumentación judicial en la referencia al principio de celeridad procesal, y en la referencia a las cuestiones del peligro en la demora (como elemento constitutivo de la medida cautelar) y a las limitaciones probatorias (en la vía judicial de amparo u otros procesos abreviados). Luego, en términos de pronóstico de salud, si bien no hay un principio expreso al respecto, consideramos que la aplicación generalizada del razonamiento por necesidades en cada uno de los casos en estudio atiende a este fin, en tanto permite rescatar las especificidades del caso concreto y, entre ellas, la urgencia vital y/o la calidad de vida del paciente.

Segundo elemento: la cartilla de servicios y prestadores, y el Programa Médico Obligatorio. Este elemento se presenta dentro de la argumentación del Tribunal como orientación respecto a las prestaciones debidas, en tanto referencia mínima incluyente de otras presta-ciones consideradas "implícitas". Se destaca la necesidad de considerar "las provisiones del PMO como condiciones mínimas, es decir, no como un techo sino como un piso prestacional, pues una interpretación reticente, contraria a la propuesta, va en desmedro de los derechos fundamentales del afiliado devenido ahora en paciente médico" (Caso F.R. c/INSSJYP, Consid. IV, Voto Dr. Jiménez).

A nuestro entender, la referencia a este elemento dentro de los casos se vislumbra a través de la aplicación del principio de legalidad y no arbitrariedad, en relación a la existencia de silencio arbitrario de la obra social o la exigencia de una negación razonablemente justificada.

Así también, se vislumbra la consideración de este elemento en la aplicación del principio de integralidad en las prestaciones de salud. A modo de ejemplo, se argumenta al respecto que "Respecto del derecho a la protección de la salud (...) debe tenerse presente que el sistema de Seguridad Social y las prestaciones derivadas del mismo, deben tender hacia la "integralidad", es decir, velar por el amparo de todas las contingencias que acechan al ser humano, entre las que se encuentran sus problemas relativos a la salud, específicamente mediante el acceso a medidas curativas, de recuperación y de rehabilitación de enfermedades. (Caso Gastón, José Luis y otro c/OSDE, Consid. V Voto Dr. Jiménez).

Tercer elemento: la condición de derechos fundamentales.

Otro de los elementos que se reitera en todos los casos es la referencia por parte del Tribunal a la condición de derechos fundamentales en relación a aquellos derechos que resultan vulnerados. En este sentido, se alude al derecho a la vida, a la salud, a la integridad psicofísica.

En este sentido, se argumenta que "El derecho a la vida -no sólo a la vida misma sino también a una buena calidad de vida y por consiguiente a una adecuada atención médica- asume un papel central en la sistemática de los derechos humanos, pues tiene por contenido un bien humano más básico que todo el resto, pues resulta ser la condición necesaria, primera y más fundamental para la realización de los otros bienes" (Caso P.A.R. y otro c/Construir Salud, Voto Dr. Ferro). 
A nuestro entender, la consideración de este tercer elemento se traduce dentro del razonamiento judicial en la aplicación del principio de dignidad humana, en términos de calidad de vida del paciente, así como también en el principio de operatividad de derechos fun-damentales (en particular, en el caso P.A.R. y otro c/Construir Salud).

\section{Cuarto elemento: la condición de vulnerabilidad o discapacidad.}

Por último, encontramos presente dentro de todos los casos en estudio la considera-ción del elemento de la vulnerabilidad o condición de discapacidad de quien reclama las prestaciones médicas. Desde el inicio, la condición de una persona enferma o con problemas de salud comprende en sí misma una vulnerabilidad propia. Aun así, entendemos que es posible traducir este elemento de vulnerabilidad en la aplicación de varios principios dentro del razonamiento judicial.

Nos interesa destacar en primer lugar, el principio de autonomía y autodeterminación del paciente. Luego, consideramos que cabe también mencionar el principio de prevalencia de la relación-médico-paciente, en términos de vínculo de confianza; ello en tanto la ruptura de ese vínculo constituye una vulnerabilidad adicional en las condiciones de un paciente.

En este sentido, se argumenta que “...no se trata aquí de otorgar livianamente una cobertura médica con un determinado profesional de la salud ante la presentación de una receta médica, sino de evitar que niños con gravísimas patologías, y acreditada condición de discapacidad, deban 'penar' administrativamente para lograr que se le provea una determinada cobertura médica con un determinado profesional, con el cual tiene una consolidada relación de confianza 'relación médico-paciente' y viene tratando su grave enfermedad con la mentada especialista" (Caso Gastón, José Luis y otro c/OSDE, Consid. VI, Voto Dr. Jimenez).

\section{Reflexiones finales}

A lo largo de este trabajo hemos intentado reconstruir el proceso de adjudicación de los jueces en cinco casos particulares en materia de salud. Compartimos con Lars Eriksson y otros teóricos de la argumentación jurídica, el criterio de insuficiencia respecto al modelo de subsunción a la hora de dar respuesta a los nuevos fenómenos y demandas sociales ${ }^{17}$.

Consideramos que la propuesta de nuevos modelos de racionalidad, de argumenta-ción o de razonamiento jurídico abre a discusión diversos tópicos dentro de la Teoría del Derecho, entre ellos la tensión entre la justicia particular, sectorial y general, y el lugar y funcio-namiento de los principios dentro del orden jurídico y de la adjudicación judicial ${ }^{18}$. Así también, entendemos en sentido inverso que evaluar los procesos de adjudicación judicial en un estudio de análisis como el que aquí se propone, apelando a los modelos de racionalidad aplicados, nos permite ir más allá de la casuística y observar la forma

\footnotetext{
17 En sintonía con este criterio de insuficiencia, Laura Clérico, Martín Aldao y Federico De Fazio (2015) analizan la jurisprudencia desarrollada por la Corte Suprema de Justicia y concluyen en la necesidad de extender la mirada judicial hacia una perspectiva integral, que comprenda aquellos factores diversos y determinantes básicos de la salud (conforme Observación General nro. 14 del Comité de Derechos Eco-nómicos, Sociales y Culturales).

18 A raíz de la aplicación del razonamiento por necesidades, uno de los elementos que claramente se pone en tensión es el de la certeza jurídica en el proceso o el valor previsibilidad. Al respecto, compartimos la respuesta de Aulis Aarnio, cuando reformula esta rigidez en un nuevo equilibrio, afirmando que "en una sociedad moderna la certeza jurídica cubre dos elementos diferentes: en el razonamiento jurídico ha de evitarse la arbitrariedad (principio del estado de derecho) y, que la decisión misma es decir el resultado final debe ser apropiado. De acuerdo con este segundo punto, las decisiones jurídicas deben estar de acuerdo no solo con el derecho formal, sino que también tienen que satisfacer ciertos criterios de certe-za axiológica (...) En la sociedad, la decisión jurídica sólo puede ser aceptable a condición de que ambos conjuntos de criterios sean satisfechos. El proceso de razonamiento debe ser racional y sus resultados deben satisfacer las demandas de justicia. Además, si una decisión no es aceptable tampoco puede ser legítima (en el sentido amplio de la palabra)" (Aarnio, 1990: p. 30).
} 
de teorizar, de concebir el derecho como objeto de estudio y como sistema jurídico por parte de los magistrados.

Según el paradigma tradicional, el recorrido del razonamiento que deriva en el fallo judicial se trata de un proceso lógico, deductivo, coherente y casi natural o determinado, operando en forma casi automática y estandarizada. Según los nuevos modelos de razonamiento, la actividad del operador jurídico hasta arribar a la decisión judicial se trata de un proceso de construcción, elaboración, y búsqueda de armonía entre el ordenamiento en su conjunto y los hechos del caso. No existiría aquí un criterio único para arribar a la sentencia, sino que se vuelve más bien una operación individualizada en relación a las características del caso.

Durante dicho proceso, el juez hace uso de una multiplicidad de herramientas, que exceden la mera lógica deductiva. A su vez, surge la posibilidad de aplicar principios allí cuando la utilización de las reglas resulta insuficiente para resolver la controversia. De este modo, el juez recurre a un razonamiento de tipo material, a fin de construir la solución más justa para el caso.

Aulis Aarnio afirma que "En un caso-situación difícil ninguna de las cadenas del silogismo da por sí misma soporte suficiente al resultado final, aunque ellas son necesarias por lo que se refiere a su fuerza justificatoria. El factor decisivo es la totalidad de los argumentos"; "Lo decisivo aquí es el grado de coherencia del conjunto de las premisas que se puede construir. La 'medida última' es así la coherencia, no la correspondencia como en las afirmaciones empíricas" (Aarnio, 1990: p. 30). Del mismo modo, en los cinco casos analizados en este trabajo hemos notado como el material probatorio y los fundamentos normativos en base a reglas jurídicas se presentan como elementos complementarios dentro del razonamiento judicial. El centro del razonamiento circula por otros espacios ${ }^{19}$.

De este modo, el juez al momento de resolver realiza un salto desde lo legalmente posible a aquello que puede determinar en el caso concreto como lo legal y valorativamente posible (y relevante).

Por último, nos interesa dejar planteado una reflexión respecto al peso de la racionalidad por necesidades en el ámbito del derecho a la salud y, por consiguiente, los obstáculos que hemos inferido a través de los casos en estudio para aplicar una racionalidad de fines o una mirada sistémica del orden jurídico por parte de la Cámara de Apelaciones.

Hemos visto como en todos los casos analizados el Tribunal culmina fundando la ma-yor parte de su hilo argumental en una racionalidad por necesidades. A nuestro entender, la proyección judicial orientada hacia los fines del sistema, dentro de la temática particular del ámbito de salud en particular, se encuentra seriamente limitada por los grandes planteos que rodean los sistemas de salud. Más allá de los tópicos tradicionales relativos a la escasez de recursos o de presupuesto, y a la extensión o restricción del alcance de la cobertura médica obligatoria, nos referimos a otro tipo de cuestiones que rozan un tinte de mayor profundidad. Aquellos interrogantes que nos ayudan a plantear límites o definiciones, tales como ¿qué comprendemos por salud?; ¿qué vamos a comprender

19 En el caso concreto, el problema mismo funciona como una fuente del derecho. Asimismo, se produce una cierta "ponderación de elementos fácticos", todos ellos vinculados al caso individual, que actúa como una de las herramientas centrales del razonamiento. De este modo, hemos visto en los casos en estudio la referencia al estado general de salud, el contexto personal y familiar, así como el contexto económico del peticionante. 
por enfermedad?; ¿cuáles son los factores de relevancia a tener en cuenta?; ¿cuáles son las vías que vamos a considerar como "legítimamente habilitadas" para la recuperación de la salud?; ¿cuál es la barrera que delimita los efectos terapéuticos y de aquellos efectos lúdicos, recreativos o de autosatisfacción en los medicamentos?; entre tantos otros. Consideramos que todos estos interrogantes constituyen las precondiciones para poder abrir el camino de la jurisprudencia hacia una adjudicación jurídica proyectada en términos de finalidades y sostenimiento del Sistema de Salud.

En suma, consideramos que los obstáculos a la aplicación de una racionalidad de fines en materia de salud encuentran su raíz en aquellos debates filosófico-jurídicos que emergen, tal y como lo indica Eriksson, con la tendencia hacia la materialización en el derecho moderno. A nuestro entender, esta línea de interrogantes que dejamos aquí planteados se perfila a futuro como uno de los grandes desafíos en el ámbito de la adjudicación judicial en materia del Derecho de la Salud.

\section{REFERENCIAS BIBLIOGRÁFICAS}

- "García Ivana R. c/ C.S. Salud s/amparo", Cámara Federal de Apelaciones de Mar del Plata, resolución del 07/05/2012. Id SAlJ: FA12390007.

- "Muñoz José Alberto c/INSSJP y otros S/ Amparo", Cámara Federal de Apela-ciones de Mar del Plata, resolución del 13/05/2014. Id SAlJ: FA14390012.

- "Gastón José Luis y otros c/OSDE Binario s/Amparo Ley", Cámara Federal de Apelaciones de Mar del Plata, resolución del 23/12/2014

- "F.R. c/INSSJYP s/Ley de Discapacidad", Cámara Federal de Apelaciones de Mar del Plata, resolución del 27/03/2015. Id. Infojus: FA 15390000.

- "P.A.R. y otro c/Construir Salud s/Ley de Incapacidad”, Cámara Federal de Apelaciones de Mar del Plata, resolución del 26/07/2017. Id SAlJ: FA17390001.

- Aarnio, Aulis (1990). La tesis de la única respuesta correcta y el principio regu-lativo del razonamiento jurídico, en Revista DOXA Cuadernos de Filosofía del Derecho, nro. 8, pp. 23-38.

- Aarnio, Aulis (1991). Lo racional como razonable. Un tratado sobre la justificación jurídica. Madrid: Centro de Estudios Constitucionales.

- Alexy, Robert (2001). Teoría de los Derechos Fundamentales, traducción de E. Garzón Valdés. Madrid: Centro de Estudios Políticos y Constitucionales. 2da. reimpresión.

- Atienza, Manuel (2005). Las razones del derecho. Teorías de la argumentación jurídica. México: Universidad Autónoma de México.

- Atienza, Manuel (2013) Curso de argumentación jurídica. Madrid: Trotta.

- Ciuro Caldani, Miguel Ángel (2005). Perspectivas trialistas para la construcción de los casos. La complejidad de los casos, en Suplemento de Actualidad La Ley, 2004-D, 1181.

- Clérico, Laura; Aldao, Martín y De Fazio, Federico (2015) “La protección del de-recho constitucional a la salud en Argentina", en Revista Gaceta Laboral, Volumen 21, nro. 3, año 2015, pp. 239-275.

- Dworkin, Ronald (1975). Hard cases, en Harvard Law Review, vol. 88, 6.

- Eriksson, Lars D. (1997). "Tendencias Conflictivas en el derecho moderno", en La normatividad del derecho, Aulis Aarnio, Ernesto Garzón Valdés y Jyrki Uusi-talo coordinadores. Madrid: Gedisa. pp. 113-126. 
- Guastini, Ricardo (2001). Estudios de Teoría Constitucional. México: UNAM.

-Waldron, Jeremy (2000). El rol de los derechos en el razonamiento práctico: 'derechos' contra 'necesidades', en Revista Argentina de Teoría Jurídica, vol 2, nro 1, Noviembre 2000, Universidad Torcuato Di Tella.

- Zagrebelsky, Gustavo (2003). El Derecho dúctil. Ley, derechos, justicia. Traduc-ción de M. Gascón. Madrid: Trotta. 5ta. Edición. 